\title{
DNA Damage Induced by Ultrasound and Cellular Responses
}

\section{Yukihiro Furusawa ${ }^{1,2^{\star}}$ and Takashi Kondo ${ }^{1}$}

${ }^{1}$ Department of Radiological Sciences, Graduate School of Medicine and Pharmaceutical Sciences, University of Toyama, Japan

${ }^{2}$ Department of Liberal Arts and Sciences, Toyama Prefectural University Toyama, Japan

\begin{abstract}
Ultrasonic technologies pervade the medical field as a long established imaging modality in clinical diagnostics and, with the emergence of targeted high-intensity focused ultrasound, as a means of thermally ablating tumors. Ultrasound (US) causes multiple thermal and non-thermal effects, such as mechanical and chemical stresses, that can result in damage to the cellular membrane and nucleus, leading to transient membrane pores, alterations in gene expression, and cell death, including apoptosis. On the basis of its biological effects US has been proposed as a new drug delivery and molecular targeting tool for cancer therapy. However, the molecular mechanisms involved in USinduced cell killing are not yet fully understood. Recently, we have reported that the mechanical effects of US elicit DNA single strand as well as double strand breaking- the most cytotoxic form of DNA damage, which initiates subsequent DNA damage response associated with DNA repair, cell cycle arrest, and cell death. Here in the present study we have focused on one of the most significant biological effects of US, i.e., DNA damage and discussed the underlying mechanisms and a unique cellular response. In addition, we have described the characteristic DNA damage response induced by heat stress, which could have caused by the thermal effects of US. Moreover, the study will enrich the literature relevant to furthering our understanding of US for future applications in cancer therapy.
\end{abstract}

Keywords: Ultrasound; DNA damage; Cell-cycle checkpoint; Cell death

\section{Introduction}

Ultrasound (US) has not only been utilized for diagnostic purposes, but also for therapy in clinical fields. In recent years, high intensity focused US (HIFU) has been developed for ultrasonic hyperthermia and thermal ablation of tumors in cancer therapy [1]. The biophysical mechanisms of US cancer therapy are classified as either thermal or non-thermal effects, the latter being further divided into cavitational and non-cavitational effects. Previously, the thermal effects of US were thought to be the principal mechanism for cancer treatment. However, subsequent studies have demonstrated negligible thermal effects for cancer treatment using low-intensity pulsed US. Non-thermal cavitation effects have also been considered to be responsible for US's potential in treating cancer. Cavitation is the formation of vapor cavities in a liquid. Cavitation is further divided into inertial cavitation and non-inertial cavitation. In aqueous solution, the collapse of cavitation bubbles produced by inertial cavitation causes the temperature and pressure of the vapor to rise, leading to the dissociation of water vapor into hydroxyl radicals and hydrogen atoms (chemical effects) and the production of mechanical stress, such as shear stress, shock wave, and high pressure (mechanical effects). All mechanisms are dependent on ultrasonic intensity and exposure time. The thermal effect is produced as a result of sound absorption and the temperature increase per unit time is proportional to the ultrasonic intensity. In addition, cavitational effects are observed only above a threshold intensity and exposure time. However, the threshold varies depending on the frequency used as well as ultrasonic fields (medium composition, tissue type, etc.) [2]. In 1987, Fecheimer et al. were the first to report the biological effects of US cavitation [3], where US was used to deliver cell impermeable fluorescent dextran molecules into mammalian cells by increasing membrane permeability, involving the mechanical production of transient membrane pores. In 1999, Ashush et al. were the first to report that US can induce apoptotic cell death in human leukemia cell lines [4], which is also demonstrated in our own research on the activation of the mitochondrial caspase pathway in US-treated leukemia cells [5]. Currently, accumulating evidence indicates that the non-thermal effects of US-induced reactive oxygen species, membrane fluidity, and DNA damage are responsible for cell death. In this review, we summarize the research on DNA damage and the cellular response provoked by US with the aim of providing information regarding the biological effects of US that may be helpful for understanding and developing its potential use in cancer therapy.

\section{US-Induced DNA Damage}

In 1963, US was shown to induce DNA base damage and digest DNA in an aqueous solution, leading to DNA single strand breaks (SSBs) as well as double strand breaks (DSBs) [6]. Today, US technology has been utilized for molecular biology techniques such as the chromatin shearing of fixed cells for chromatin immunoprecipitation assays, and DNA shearing for library construction in next generation sequencing [7,8]. In terms of DNA damage in living cells exposed to US, sister chromatid exchange in human lymphocytes, base damage in mouse breast cancer EMT6 cells, and SSB formation in human lymphocyte and Chinese hamster ovarian $(\mathrm{CHO})$ cells have previously been phosphorylation of histone $\mathrm{H} 2 \mathrm{AX}$ on Ser139 $(\gamma \mathrm{H} 2 \mathrm{AX})$, a sensitive biomarker for DSBs (detailed in the following section). The presence of DSBs was further confirmed using a single cell gel electrophoresis assay, called the neutral comet assay, a sensitive method for quantifying DSBs at the single cell level. One of the characteristics of US-induced DSBs is the heterogeneity of $\mathrm{H} 2 \mathrm{AX}$ phosphorylation within the population of cells, which contrasts with the homogeneity of $\mathrm{H} 2 \mathrm{AX}$ phosphorylation in cells irradiated with ionizing radiation (IR) reported (Figure 1) [4,9-11]. Suppression of inertial cavitation by triatomic $\mathrm{N}_{2} \mathrm{O}$ gas or addition of a free radical scavenger has been shown to almost completely diminish or partially attenuate SSB formation, respectively [5]. This indicates that US-induced DNA damage is largely dependent on mechanical stress, and to a lesser degree, on free radicals that are induced by inertial cavitation.

*Corresponding author: Yukihiro Furusawa, Department of Liberal Arts and Sciences, Toyama Prefectural University, 5180, Kurokawa, Toyama 939-0398, Japan, Tel: +81-766-56-7500; Fax: +81-766-56-6117; E-mail: furusawa@pu-toyama.ac.jp

Received February 20, 2017; Accepted March 03, 2017; Published March 10, 2017

Citation: Furusawa Y, Kondo T (2017) DNA Damage Induced by Ultrasound and Cellular Responses. Mol Biol 6: 188. doi: 10.4172/2168-9547.1000188

Copyright: (c) 2017 Furusawa Y, et al. This is an open-access article distributed under the terms of the Creative Commons Attribution License, which permits unrestricted use, distribution, and reproduction in any medium, provided the original author and source are credited. 

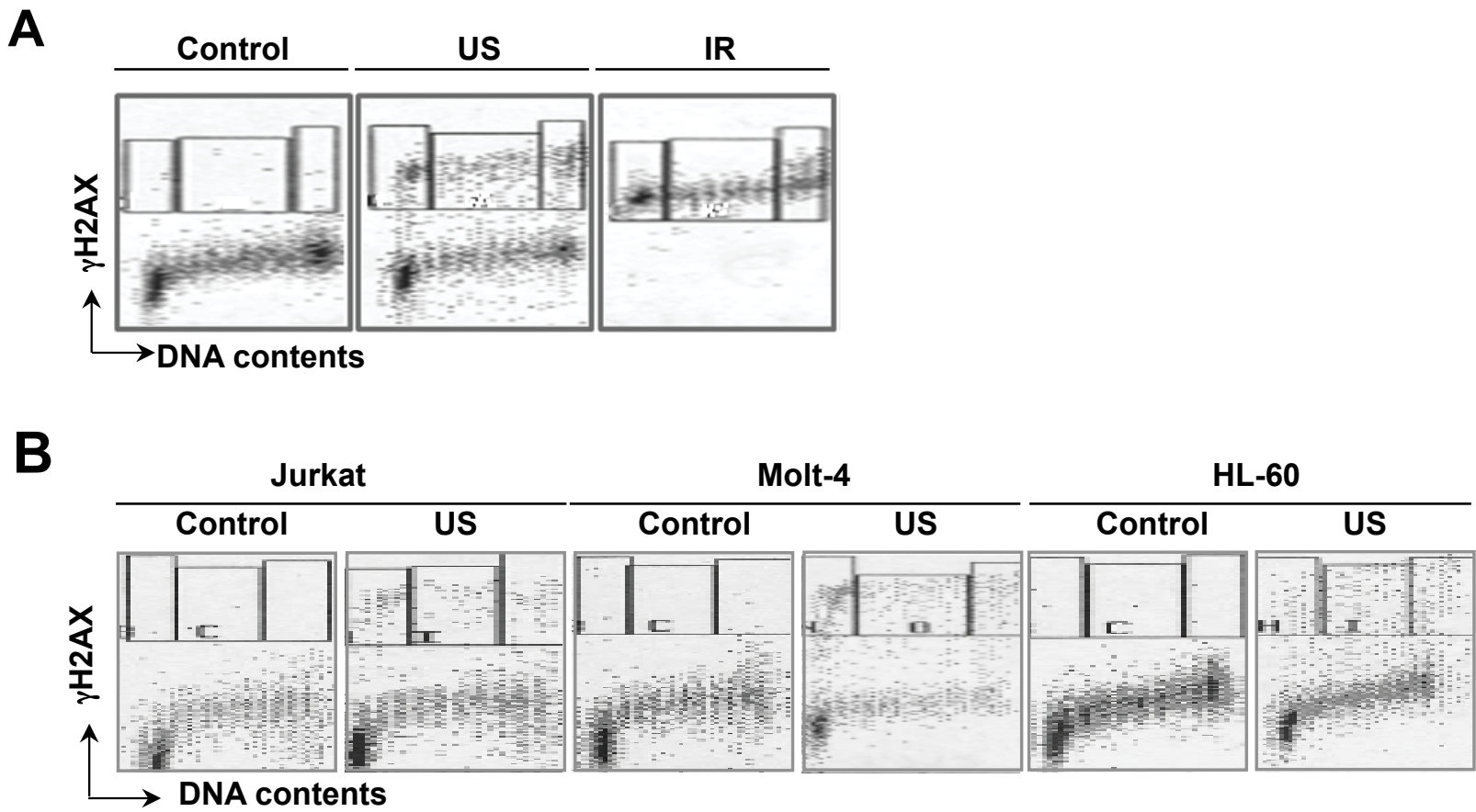

Figure 1: Distribution of $\mathrm{YH} 2 \mathrm{AX}$ content and DNA content in cells exposed to US $\left(0.3 \mathrm{~W} / \mathrm{cm}^{2}\right)$ or IR (10 Gy). After a 30 min incubation period, cells were collected and stained as described in [12]. (A) Representative histograms in control, US and IR-exposed U937 cells (B) Distribution of yH2AX positive cells in Jurkat, Molt-4, and $\mathrm{HL}-60$ cells post-US exposure.

Despite the accumulating evidence for US-induced SSBs, there have been no report showing the US-induced DSBs in the cell nucleus, which is the most cytotoxic form of DNA damage. This is probably due to the use of classical and relatively insensitive methods for DSB detection (e.g., neutral elution assays and pulse field gel electrophoresis) because these methods require a 50-100 Gy radiation dose to clearly detect the presence of DSBs in the cell nucleus. In recent years, we were the first to detect US-induced DSBs in the cell nucleus by using a highly sensitive method for DSB detection [12]. In human leukemia cells exposed to US, we detected phosphorylation of histone H2AX on Ser139 $(\gamma \mathrm{H} 2 \mathrm{AX})$, a sensitive biomarker for DSBs (detailed in the following section). The presence of DSBs was further confirmed using a single cell gel electrophoresis assay, called the neutral comet assay, a sensitive method for quantifying DSBs at the single cell level. One of the characteristics of US-induced DSBs is the heterogeneity of H2AX phosphorylation within the population of cells, which contrasts with the homogeneity of H2AX phosphorylation in cells irradiated with ionizing radiation (IR) (Figure 1).

\section{US-Induced DSB Formation Mechanism}

The difference in $\gamma \mathrm{H} 2 \mathrm{AX}$ staining patterns between US- and IRexposed cells may reflect the different mechanisms underlying DSB formation. IR-induced DSBs are attributed directly to DNA ionization, and indirectly to free radicals. In the case of US, cavitational effects are responsible for DSB induction because suppression of inertial cavitation by $\mathrm{N}_{2} \mathrm{O}$ gas completely diminished the induction of $\gamma \mathrm{H} 2 \mathrm{AX}$ positive cells (Figure 2). As described above, cavitation effects are further classified as either chemical or mechanical effects. Considering that both IR and US have the potential to induce free radical formation, it seems plausible to expect the involvement of free radicals in USinduced DSBs. However, in contrast with SSB formation, addition of free radical scavengers, such as DMSO or $\mathrm{N}$-acetyl cysteine, hardly affect
US-induced $\gamma \mathrm{H} 2 \mathrm{AX}$ levels despite the suppression of extracellular and intracellular hydroxyl radical formation. Therefore, it is reasonable to assume that the mechanical effects of US cavitation, such as shear stress, are more essential for DSB formation in cells exposed to US. Consistent with this observation, our classical study in 1985 demonstrated that DSB induction in naked DNA is caused by the mechanical effects of US but not chemical effects [13]. However, from the available literature, there is no direct evidence showing a correlation between such mechanical effects and DSB formation in the cell nucleus.

\section{Phosphorylation of Histone H2ax in Cells Exposed to US}

$\gamma \mathrm{H} 2 \mathrm{AX}$ is a phosphorylated histone $\mathrm{H} 2$ variant that was reported to be a sensitive biomarker for the presence of DSBs [14]. In the presence of DSBs, DNA damage sensor proteins such as Ataxia telangiectasia mutated (ATM), Ataxia telangiectasia mutated and Rad3-related (ATR) and DNA-dependent protein kinase (DNA-PK), members of the phosphatidylinositol 3-kinase-related kinase (PIKKs) family, are known to phosphorylate histone H2AX [15]. In cells exposed to IR or DNA-damaging agents such as bleomycin, H2AX phosphorylation is generally dependent on ATM, or ATR and DNA-PK in the absence of ATM [16]. In the presence of replicative stress by hydroxyurea, H2AX phosphorylation is largely dependent on ATR and is restricted in cells during the $S$ phase [17]. Additionally, cells receiving apoptotic signaling showed H2AX phosphorylation that is DNA-PK dependent [18], which was further corroborated in experiments using DNA-PK inhibitor and TNF-related apoptosis-inducing ligand (TRAIL) [18]. In line with previous research, we reported a reduction in the IRinduced $\gamma \mathrm{H} 2 \mathrm{AX}$ positive cell population in cells with an ATM specific inhibitor (KU55933) [12]. In the absence of ATM inhibitor, DNA-PK specific inhibitor (NU7026) did not affected the population of $\gamma \mathrm{H} 2 \mathrm{AX}$ positive cell, indicating that ATM is predominant kinase involved in 


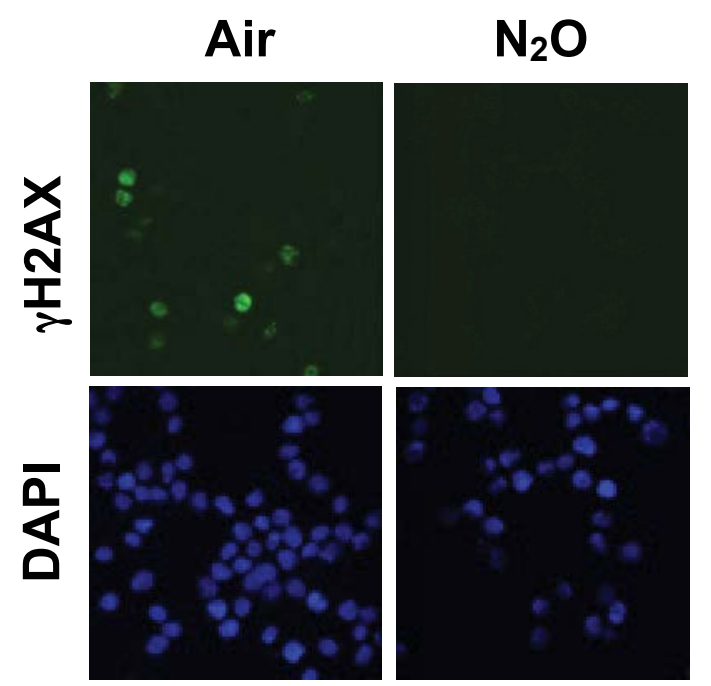

Figure 2: The effect of triatomic $\mathrm{N}_{2} \mathrm{O}$ gas on the induction of $\mathrm{yH} 2 \mathrm{AX}$. U937 cells were suspended in culture medium saturated with air or $\mathrm{N}_{2} \mathrm{O}$ followed by sonication. $\mathrm{N}_{2} \mathrm{O}$ saturation and $\mathrm{y} \mathrm{H} 2 \mathrm{AX}$ staining were performed as described in [12].

H2AX phosphorylation after IR-exposure. However, interestingly DNA-PK inhibitor markedly suppressed H2AX phosphorylation induced by US, In addition, combination of the ATM and DNA-PK inhibitors almost diminished the H2AX phosphorylation. These results indicate that $\gamma \mathrm{H} 2 \mathrm{AX}$ induced by US is dependent on DNA-PK rather than ATM, in contrast with that induced by IR. In agreement with these results, US preferentially phosphorylates DNA-PK at Ser2056 over ATM at Ser1981, the auto-phosphorylation sites of these kinases. Additionally, in support of the lack of correlation between ATR and $\mathrm{H} 2 \mathrm{AX}$ phosphorylation, US-induced $\gamma \mathrm{H} 2 \mathrm{AX}$ positive cells were not restricted during the $\mathrm{S}$ phase (Figure 1). DNA-PK-dependent H2AX phosphorylation was initially thought to be associated with apoptotic signaling [18]. However, in contrast with treatment with TRAIL, pretreatment with Z-VAD FMK, a pan-caspase inhibitor, hardly affected the tail moment and population of $\gamma \mathrm{H} 2 \mathrm{AX}$ positive cells when exposed to US, indicating that US-induced $\gamma \mathrm{H} 2 \mathrm{AX}$ reflects DNA damage but not initial apoptotic signaling. As observed in the $\gamma \mathrm{H} 2 \mathrm{AX}$ staining patterns, IR-treated cells showed staining at discrete foci, whereas TRAIL-treated cells showed peri-nuclear (peripheral), pan-nuclear (diffuse), and apoptotic body localized staining (Figure 3). In contrast, US-treated cells showed discrete foci, or pan-nuclear staining with discrete foci. In summary, preferential DNA-PK activation followed by $\gamma \mathrm{H} 2 \mathrm{AX}$ phosphorylation and unique $\gamma \mathrm{H} 2 \mathrm{AX}$ staining patterns are features of the DNA damage response induced by US. However, the H2AX phosphorylation by DNA-PK remains unclear.

\section{US-induced DSBs and the Cell Death Signaling Pathway}

PIKK family proteins are kinases that phosphorylate H2AX as well as many effector molecules that regulate DNA repair, cell death and cell survival [20-22]. In the presence of DNA damage, ATM and ATR are known to activate checkpoint kinase and p53 to regulate the cellcycle checkpoint, cell survival, and apoptosis. Additionally, ATM and DNA-PK promote cell survival through Akt phosphorylation at Ser473 [23]. In 2004, Abdollahi et al. reported the molecular mechanisms underlying p53-dependent apoptotic signaling in cells exposed to US. They demonstrated that TK6 lymphocyte cells with p53 defects were more resistant to US than parental control cells harboring wild-type p53 [24], suggesting a pivotal role for p53 in US-induced cell death signaling. Consistent with this report, we also confirmed that Molt4 cells (human leukemic lymphoblast cells) stably transfected with shRNA targeting p53 (Molt-4/shp53) were more resistant to USinduced apoptosis than parental control cells [25]. Furthermore, we found that p53 phosphorylation at Ser15 in cells exposed to US was dependent on ATM rather than DNA-PK (Figure 4). On the other hand, Akt phosphorylation was dependent on DNA-PK rather than ATM, which was independent of the p53 phenotype. Consistent with this difference between Akt and p53 phosphorylation, ATM inhibitor KU55933 attenuated cleavage of caspase- 3 and slightly suppressed US-induced cell death in Molt-4 cells, but not in Molt-4/shp53 cells. Moreover, the DNA-PK inhibitor NU7026 promoted US-induced caspase- 3 cleavage and cell death in both cell types, indicating that the ATM-p53 and DNA-PK-Akt axes play opposite roles in US-induced cell death, including apoptosis. Moreover, these results suggest that DNAPK may be a useful as a molecular target for US-aided tumor therapy, regardless of the $\mathrm{p} 53$ phenotype.

\section{DNA Repair of US Induced DSBs}

$\gamma \mathrm{H} 2 \mathrm{AX}$ forms a platform for DNA repair by recruiting and maintaining DNA repair proteins. ATM is recruited to DSB sites in conjunction with the MRE11/RAD50/NBS1 (MRN) complex that recognizes the double-stranded end of DNA and contributes to DNA repair, as found in homologous recombination [26]. DNA-PK binds to the $\mathrm{Ku} 70 / \mathrm{Ku} 80$ heterodimer that binds to and protects DNA ends from degradation, and contributes to DNA repair, such as nonhomologous end joining [27]. In addition to $\gamma \mathrm{H} 2 \mathrm{AX}$ foci formation, nuclear localization of phospho-ATM at Ser1981, phospho-DNA-PK at Ser2056/T2609 and phosphor-NBS1 at Ser343, which are activated forms of these proteins [28], were observed in US-treated cells [12].

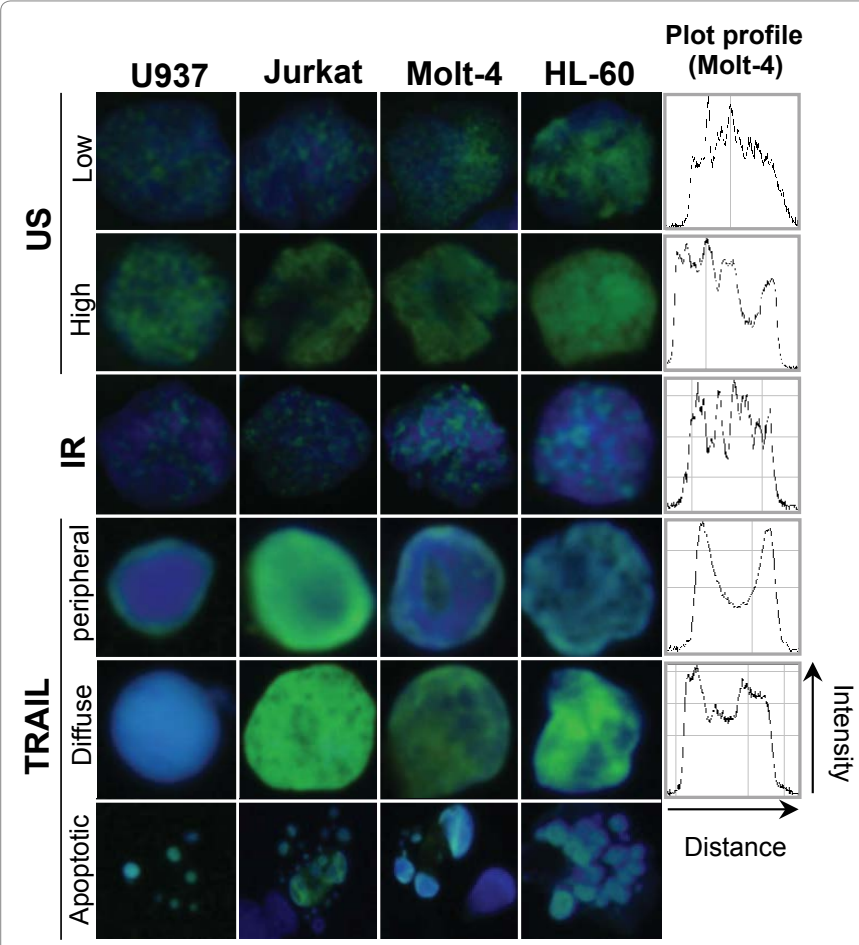

Figure 3: Immunofluorescence analysis of $\mathrm{yH} 2 \mathrm{AX}$ in U937, Jurkat, Molt-4, and $\mathrm{HL}-60$ cells with US, IR ( $3 \mathrm{~Gy})$, or TRAIL $(0.1 \mathrm{mg} / \mathrm{mL})$. $\mathrm{YH} 2 \mathrm{AX}$ staining was performed as described in [12]. Plot profiles of $\mathrm{yH} 2 \mathrm{AX}$ intensity in Molt-4 cells were obtained by using $\mathrm{NIH}$ Image $\mathrm{J}$ software. 


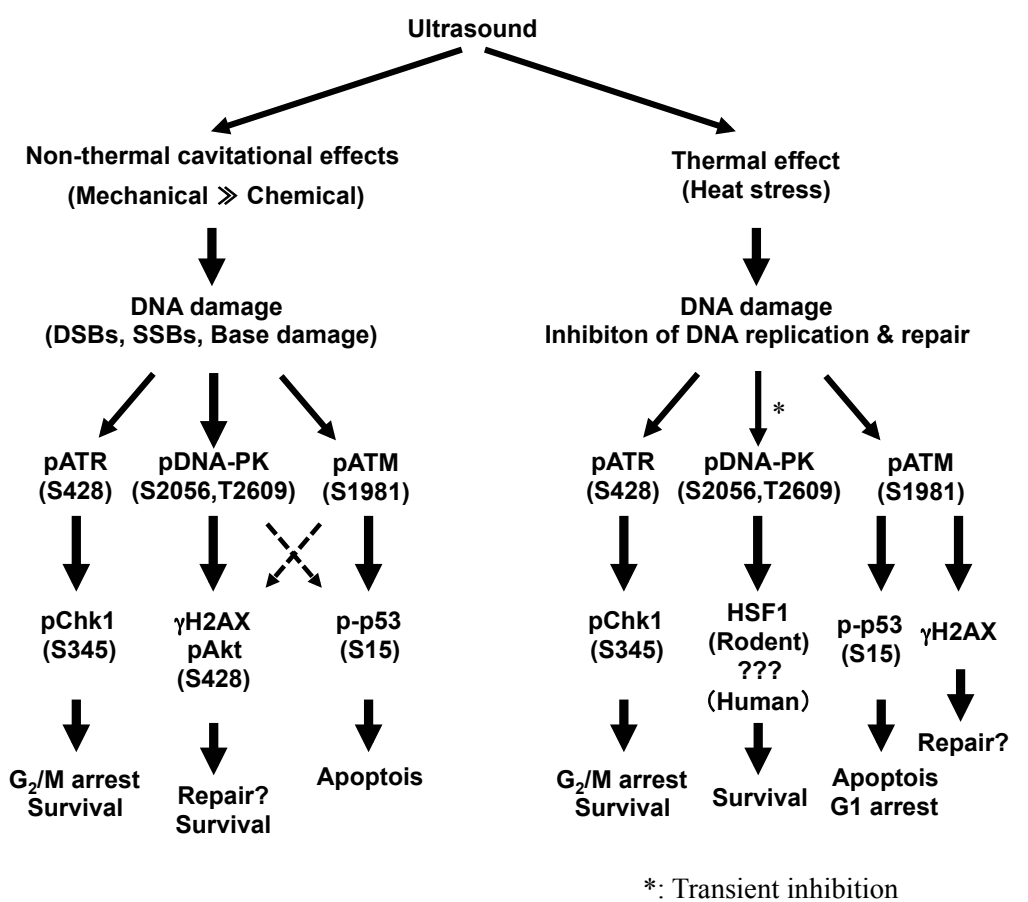

Figure 4: US and HS- induced DNA damage and signal transduction associated with cell-cycle arrest, cell survival, and apoptosis.

Considering that both $\gamma \mathrm{H} 2 \mathrm{AX}$ intensity and tail moment were attenuated in a time-dependent manner post US-exposure, it is possible that these molecules contribute to DNA repair. However, to the best of our knowledge, there is not yet direct evidence demonstrating the role of these molecules in US-induced DSB repair. It should be further investigated if these proteins contribute to DNA repair in cells with USinduced DSBs.

\section{US Induced DNA Damage and Cell Cycle Checkpoint}

Cells exposed to genotoxic stress activate cell-cycle checkpoint machinery to negatively regulate cell cycle progression. A cell with DNA damage avoids entering the next cell-cycle phase until the DNA damage is repaired in order to promote cell survival. The cell-cycle checkpoint is composed of $G_{1}, S$, and $G_{2} / M$ checkpoint [29], where the mechanism of action of the intra-S phase checkpoint is less clear than that of the $\mathrm{G}_{1}$ and $\mathrm{G}_{2} / \mathrm{M}$ phase checkpoints [30,31]. The $\mathrm{G}_{1}$ checkpoint is mainly regulated by cyclin dependent kinase $1 \mathrm{~A}$ (coding $\mathrm{p} 21$ ), which is a representative transcript of 553 [32]. The $\mathrm{G}_{2} / \mathrm{M}$ checkpoint is regulated by checkpoint kinase 1 and 2 (Chk1 and Chk2), which are downstream of ATM and ATR [29,33]. Chk1 and Chk2, serine/threonine kinases that are functional analogs with dissimilar structures, contribute to cellcycle arrest by phosphorylating CDC25 phosphatases.

Since $\mathrm{G}_{1}$ checkpoint activation by $\mathrm{p} 21$ is attenuated in cells with p53 defects, the cells rely on Chk1/2 and the $G_{2} / M$ checkpoint for cell survival when DNA damaging agents are encountered. Considering that at least half of all tumors exhibit a p53 mutation or deletion, selective inhibition of Chk $1 / 2$ is a promising strategy for cancer therapy, and some selective Chk1/2 inhibitors are currently in preclinical or phase I/ II clinical trials (shown in https://clinicaltrials.gov) [34-36].

In 2011, Zhong et al. reported US-induced apoptosis and cellcycle arrest at the $\mathrm{G}_{2} / \mathrm{M}$ phase in human leukemia HL-60 cells [37]. Our subsequent research revealed that US-induced $G_{2} / M$ arrest is dependent on Chk1 in human leukemia Jurkat cells [38]. In general,
ATM preferentially phosphorylates Chk2 at Thr68 over Chk1 at Ser345, and Chk1 phosphorylation is largely dependent on ATR [39]. Consistent with previous reports, US-induced Chk1 phosphorylation was attenuated by selective inhibitors against ATR (described in [40]), but not ATM inhibitors. Chk1 inhibition using the selective inhibitor SB218078, or Chk1-targeted siRNA transfection, decreased the population of cells in the $\mathrm{G}_{2} / \mathrm{M}$ phase and increased that in $\mathrm{SubG}_{1}$ phase following US-exposure, indicating that Chk1 plays an essential role in the $G_{2} / M$ arrest and cell survival in response to US-induced DNA damage. Therefore, Chk1 may be a candidate molecular target for USaided cancer therapy. Meanwhile, the role of Chk2, a functional analog of Chk1, in US-induced cell-cycle arrest and cell death has not yet been elucidated. Considering that both ATM and Chk2 have the potential to phosphorylate and stabilize p53 (at Ser15 and Ser20, respectively), the ATM-Chk2 axis might contribute to p53-dependent apoptosis or p21-dependent $G_{1}$ checkpoint activation in cells exposed to US. Further research investigating the role of ATM-Chk2 in US-induced cell death and cell-cycle arrest is currently underway.

\section{Heat Induced DNA Damage and $\gamma \mathrm{H} 2 \mathrm{AX}$}

In the previous sections, we described the DNA damage and cellular response induced by the non-thermal effects of US. Seeing as US has been utilized for hyperthermic cancer therapy and the thermal ablation of tumors, the understanding of heat-induced DNA damage and the associated cellular response may be useful for US applications in medical fields. Many excellent textbooks and reviews describing DNA damage induced by heat stress (HS) have been published [4143]. For the purpose of this review, we will briefly describe the features of heat-induced DNA damage and the associated cellular response. Regarding the types of DNA damage observed following heat stress (HS), DNA SSBs, base damage, inhibition of DNA replication, and DNA repair have been reported [44]. The main mechanisms underlying heat-induced DNA damage are the production of free radicals and the denaturation of proteins associated with DNA replication and repair. 
HS also induces $\gamma \mathrm{H} 2 \mathrm{AX}$ foci; however, there are characteristic features of $\gamma \mathrm{H} 2 \mathrm{AX}$ foci resulting from the heat-induced DNA damage response. Firstly, heat-induced $\gamma \mathrm{H} 2 \mathrm{AX}$ foci are mainly observed in, but not restricted to, cells in the S phase [44]. Secondly, H2AX phosphorylation induced by HS is dependent on ATM but not on ATR and DNA-PK, which is different to $\gamma \mathrm{H} 2 \mathrm{AX}$ induced by hydroxyurea and US [45]. Lastly, 53BP1, a DNA repair protein also utilized as a biomarker for DSBs, does not form foci in the cell nucleus under HS [46]. This can be explained by 53BP1 having a high molecular weight $(\sim 220 \mathrm{kDa}$ as a monomer and $450 \mathrm{kDa}$ as a dimer) and is therefore sensitive to thermal denaturation. In fact, DNA-PK $(470 \mathrm{kDa})$ is also sensitive to HS and transiently loses its activity under HS, although its activity recovers in a time-dependent manner post HS treatment, probably due to the induction of molecular chaperones such as heat shock proteins [47]. It should therefore be considered that protein denaturation might affect the function of molecules in the canonical DNA damage response pathway.

\section{Heat Induced DNA Damage and Cell Death}

p53 has a long-established and well-characterized role in heatinduced apoptosis. As is the case for IR- and US-treated cells, cells harboring wild-type p53 are more sensitive to HS than parental controls $[48,49]$. Phosphorylation of $\mathrm{p} 53$ at Ser15 is dependent on ATM even in cells exposed to HS [50], which contributes to the stability of $\mathrm{p} 53$ through the dissociation of MDM2. In contrast with the role of p53 in HS-induced cell death, that of ATM and DNA-PK is more controversial. Mouse embryonic fibroblasts (MEF) with defects in ATM production showed high sensitivity to HS in comparison with parental controls [51]. Conversely, sensitivity to HS in fibroblasts isolated from ataxia-telangiectasia patients harboring mutated ATM is comparable to that in normal fibroblasts [51]. In addition, rodent cells defected with DNA-PK showed increased heat sensitivity due to the inhibition of heat shock factor 1 (HSF1) [52]; however, the contribution of DNA-PK to HSF1 activation was not confirmed in human ovarian cell carcinoma HeLa cells [53]. It is possible that the role of ATM and DNA-PK in heat-induced cell death is different between mammalian cell types and is perhaps species dependent. The abovementioned findings may be useful in evaluating the molecular target for hyperthermia or in considering the use of HS for cancer therapy.

\section{Heat Induced DNA Damage and Cell Cycle Checkpoints}

Accumulating evidence has shown that $\mathrm{HS}$ can cause $\mathrm{G}_{1}$ and $\mathrm{G}_{2} / \mathrm{M}$ arrest [54-56] p53-dependent transcription of p21 and p38 MAPK dependent CDC25A degradation were demonstrated as mechanisms for HS-induced $G_{1}$ arrest [57]. However, the molecular mechanism underlying $G_{2} / M$ checkpoint activation in cells exposed to $H S$ had not been extensively investigated. Recently, we demonstrated that HS activates the ATR-Chk1 pathway, resulting in $\mathrm{G}_{2} / \mathrm{M}$ checkpoint activation and apoptosis evasion in human leukemia Jurkat cells and several adherent cancer cells (HeLa, human squamous cell carcinoma HSC-3, and prostate cancer PC3 cells) [57]. The detailed mechanisms by which HS activates ATR have yet to be elucidated h however, molecules in the canonical ATR pathway, such as Rad9, Rad17, topoisomerase (DNA) II binding protein 1 (TopBP1), and claspin, seem to be important for ATR activation under HS [59].

\section{Conclusion}

Exhaustive studies of the DNA damage response pathway have been developed by evaluating the cellular response to IR and chemotherapeutic agents. In recent years, inhibition of the DNA damage response pathway by small molecules, particularly inhibition of DNA repair or cell-cycle checkpoint activation (e.g., PARP inhibitor and Chk1/2 inhibitor), is currently being investigated as an approach to enhance the effects of DNA damaging agents in clinical cancer therapy [60]. In addition to the application of US for thermal ablation of tumors and hyperthermia in clinical fields, research on the use of US as a tool for drug and gene delivery has also been published [61]. Therefore, the use of US for drug delivery might offer a dual advantage for cancer therapy since US has potential to both induce DNA damage, and deliver the drug or gene targeting the DNA damage response pathway. Many studies, including ours, were performed in human cultured cells; however, further study in vivo is needed for the further development and clinical application of US-aided cancer therapy.

\section{Conflict of Interest}

No conflict of interest to disclose.

\section{Acknowledgement}

This work was supported in part by Uehara Memorial Foundation, Tamura Science and Technology Foundation.

\section{References}

1. Hsiao YH, Kuo SJ, Tsai HD, Chou MC, Yeh GP, et al. (2016) Clinical Application of High-intensity Focused Ultrasound in Cancer Therapy. J Cancer 7: 225-231.

2. Kondo T, Grieser F, Choi PK, Enomoto N, Harada H, et al. (2015) Application of Ultrasound in Medicine and Biotechnology" In "Sonochemistry and the Acoustic Bubble" pp: 207-230, Elsevier Inc, New York.

3. Fechheimer M, Boylan JF, Parker S, Sisken JE, Patel GL, et al. (1987) Transfection of mammalian cells with plasmid DNA by scrape loading and sonication loading. Proc Natl Acad Sci 84: 8463-8467.

4. Ashush H, Rozenszajn LA, Blass M, Barda-Saad M, Azimov D, et al. (2000) Apoptosis induction of human myeloid leukemic cells by ultrasound exposure. Cancer Res 60: 1014-1020.

5. Honda H, Zhao QL, Kondo T (2002) Effects of dissolved gases and an echo contrast agent on apoptosis induced by ultrasound and its mechanism via the mitochondria-caspase pathway. Ultrasound Med Biol 28: 673-682.

6. Fuciarelli AF, Sisk EC, Thomas RM, Miller DL (1995) Induction of base damage in DNA solutions by ultrasonic cavitation. Free Radic Biol Med 18: 231-238.

7. Furusawa Y, Obata Y, Fukuda S, Endo TA, Nakato G, et al. (2013) Commensal microbe-derived butyrate induces the differentiation of colonic regulatory $T$ cells. Nature 504: 446-450.

8. Obata Y, Furusawa Y, Endo TA, Sharif J, Takahashi D, et al. (2014) The epigenetic regulator Uhrf1 facilitates the proliferation and maturation of colonic regulatory T cells. Nat Immunol 15: 571-579.

9. Miller DL, Reese JA, Frazier ME (1989) Single strand DNA breaks in human leukocytes induced by ultrasound in vitro. Ultrasound Med Biol 15: 765-771.

10. Miller DL, Thomas RM, Frazier ME (1991) Single strand breaks in $\mathrm{CHO}$ cell DNA induced by ultrasonic cavitation in vitro. Ultrasound Med Biol 17: 401-406.

11. Miller DL, Thomas RM, Buschbom RL (1995) Comet assay reveals DNA strand breaks induced by ultrasonic cavitation in vitro. Ultrasound Med Biol 21: 841848

12. Furusawa $Y$, Fujiwara $Y$, Campbell $P$, Zhao QL, Ogawa R, et al. (2012) DNA double-strand breaks induced by cavitational mechanical effects of ultrasound in cancer cell lines. PLoS One 7: e29012.

13. Kondo T, Arai S, Kuwabara M, Yoshii G, Kano E (1985) Damage in DNA irradiated with $1.2 \mathrm{MHz}$ ultrasound and its effect on template activity of DNA for RNA synthesis. Radiat Res 104: 284-292.

14. Rogakou EP, Boon C, Redon C, Bonner WM (1999) Megabase chromatin domains involved in DNA double-strand breaks in vivo. J Cell Biol 146: 905-916.

15. Bonner WM, Redon CE, Dickey JS, Nakamura AJ, Sedelnikova OA, et al. (2008) GammaH2AX and cancer. Nat Rev Cancer 8: 957-967.

16. Stiff T, O'Driscoll M, Rief N, Iwabuchi K, Löbrich M, et al. (2004) ATM and DNAPK function redundantly to phosphorylate $\mathrm{H} 2 \mathrm{AX}$ after exposure to ionizing radiation. Cancer Res 64: 2390-2396. 
17. Ward IM, Chen J (2001) Histone H2AX is phosphorylated in an ATR-dependent manner in response to replicational stress. J Biol Chem 276: 47759-47762.

18. Mukherjee B, Kessinger C, Kobayashi J, Chen BP, Chen DJ, et al. (2006) DNA-PK phosphorylates histone $\mathrm{H} 2 \mathrm{AX}$ during apoptotic DNA fragmentation in mammalian cells. DNA Repair (Amst) 5: 575-590.

19. Solier S, Sordet O, Kohn KW, Pommier Y (2009) Death receptor-induced activation of the Chk2- and histone $\mathrm{H} 2 \mathrm{AX}$-associated DNA damage response pathways. Mol Cell Biol 29: 68-82.

20. Jackson SP, Bartek J (2009) The DNA-damage response in human biology and disease. Nature 461: 1071-1078.

21. Lavin MF, Kozlov S (2007) ATM activation and DNA damage response. Cell Cycle 6: 931-942.

22. Harper JW, Elledge SJ (2007) The DNA damage response: Ten years after. Mo Cell 28: 739-745.

23. Bozulic L, Surucu B, Hynx D, Hemmings BA (2008) PKBalpha/Akt1 acts downstream of DNA-PK in the DNA double-strand break response and promotes survival. Mol Cell 30: 203-213.

24. Abdollahi A, Domhan S, Jenne JW, Hallaj M, Dell'Aqua G, et al. (2004) Apoptosis signals in lymphoblasts induced by focused ultrasound. FASEB $J$ 18: $1413-1414$.

25. Furusawa Y, Fujiwara Y, Hassan MA, Tabuchi Y, Morita A, et al. (2012) Inhibition of DNA-dependent protein kinase promotes ultrasound-induced cell death including apoptosis in human leukemia cells. Cancer Lett 322: 107-112.

26. Lee JH, Paull TT (2005) ATM activation by DNA double-strand breaks through the Mre11-Rad50-Nbs1 complex. Science 308: 551-554.

27. Jeggo PA (1998) DNA breakage and repair. Adv Genet 38: 185-218.

28. Takahashi A, Mori E, Ohnishi T (2010) The foci of DNA double strand break recognition proteins localize with gammaH2AX after heat treatment. J Radiat Res 51: 91-95.

29. Kastan MB, Bartek J (2004) Cell-cycle checkpoints and cancer. Nature 432 316-323.

30. Seiler JA, Conti C, Syed A, Aladjem MI, Pommier Y (2007) The Intra-S-Phase Checkpoint Affects both the DNA Replication Initiation and Elongation: SingleCell and DNA Fibre Analyses. Mol Cell Biol 27: 5806-5818.

31. Sørensen CS, Syljuåsen RG, Falck J, Schroeder T, Rönnstrand L, et al. (2003) Chk1 regulates the $S$ phase checkpoint by coupling the physiological turnover and ionizing radiation-induced accelerated proteolysis of Cdc25A. Cancer Cell 3: $247-58$.

32. Abbas T, Dutta A (2009) p21 in cancer: intricate networks and multiple activities. Nature Reviews Cancer 9: 400-414

33. Bucher N, Britten CD (2008) G2 checkpoint abrogation and checkpoint kinase-1 targeting in the treatment of cancer. $\mathrm{Br} \mathrm{J}$ Cancer 98: 523-528.

34. Walton MI, Eve PD, Hayes A, Valenti M, De Haven Brandon A, et al. (2010) The preclinical pharmacology and therapeutic activity of the novel CHK1 inhibitor SAR-020106. Mol Cancer Ther 9: 89-100.

35. Walton MI, Eve PD, Hayes A, Valenti MR, De Haven Brandon AK, et al. (2012) CCT244747 is a novel potent and selective CHK1 inhibitor with oral efficacy alone and in combination with genotoxic anticancer drugs. Clin Cancer Res 18: $5650-5661$

36. Syljuåsen RG, Sorensen CS, Nylandsted J, Lukas C, Lukas J, et al. (2004) Inhibition of Chk1 by CEP-3891 accelerates mitotic nuclear fragmentation in response to ionizing Radiation. Cancer Res 64: 9035-9040

37. Zhong W, Sit WH, Wan JMF, Yu ACH (2011) Sonoporation induces apoptosis and cell cycle arrest in human promyelocytic leukemia cells. Ultrasound Med Biol 37: 2149-2159.

38. Furusawa $Y$, lizumi T, Fujiwara $Y$, Hassan MA, Tabuchi $Y$, et al. (2012) Ultrasound activates ataxia telangiectasia mutated- and rad3-related (ATR) checkpoint kinase 1 (Chk1) pathway in human leukemia Jurkat cells. Ultrason Sonochem 19: 1246-1251.

39. Dai Y, Grant S (2010) New insights into checkpoint kinase 1 in the DNA damage response signaling network. Clin Cancer Res 16: 376-383.

40. Nishida H, Tatewaki N, Nakajima Y, Magara T, Ko KM, et al. (2009) Inhibition of ATR protein kinase activity by schisandrin B in DNA damage response. Nucleic Acids Res 37: 5678-5689.
41. Takahashi A Ohnishi T (2005) Does gammaH2AX foci formation depend on the presence of DNA double strand breaks? Cancer Lett 229: 171-179.

42. Kantidze OL, Velichko AK, Luzhin AV, Razin SV (2016) Heat Stress-Induced DNA Damage. Acta Naturae 8: 75-78

43. Kokura S, Yoshikawa T, Ohnishi T (2016) Hyperthermic Oncology from Bench to Bedside. Springer, Singapore.

44. Takahashi A, Matsumoto H, Nagayama K, Kitano M, Hirose S, et al. (2004) Evidence for the involvement of double-strand breaks in heat-induced cell killing. Cancer Res 64: 8839-8845.

45. Takahashi A, Mori E, Su X, Nakagawa Y, Okamoto N, et al. (2010) ATM is the predominant kinase involved in the phosphorylation of histone $\mathrm{H} 2 \mathrm{AX}$ after heating. J Radiat Res 51: 417-422.

46. Hunt CR, Pandita RK, Laszlo A Higashikubo R, Agarwal M, et al. (2007) Hyperthermia activates a subset of ataxia-telangiectasia mutated effectors independent of DNA strand breaks and heat shock protein 70 status. Cancer Res 67: 3010-3017.

47. Umeda N, Matsumoto Y, Yin H-L, Tomita M, Enomoto A, et al. (2003) Difference in the heat sensitivity of DNA-dependent protein kinase activity among mouse, hamster and human cells. Int J Radiat Biol 79: 671-680.

48. Ohnishi K, Ohnishi T (2001) Heat-induced p53-dependent signal transduction and its role in hyperthermic cancer therapy. Int J Hyperthermia 17: 415-427.

49. Ohnishi T (2005) The role of the p53 molecule in cancer therapies with radiation and/or hyperthermia. J Cancer Res Ther 1: 147-150.

50. Miyakoda M, Suzuki K, Kodama S, Watanabe M (2002) Activation of ATM and phosphorylation of p53 by heat shock. Oncogene 21: 1090-1096.

51. Tomita M (2010) Involvement of DNA-PK and ATM in radiation- and heatinduced DNA damage recognition and apoptotic cell death. J Radiat Res 51 : 493-501.

52. Nueda A, Hudson F, Mivechi NF, Dynan WS (1999) DNA-dependent protein kinase protects against heat-induced apoptosis. J Biol Chem 274: 1498814996.

53. Okazawa S, Furusawa Y, Kariya A, Hassan MA, Arai M, et al. (2013) Inactivation of DNA-dependent protein kinase promotes heat-induced apoptosis independently of heat-shock protein induction in human cancer cell lines. PLoS One 8: e58325.

54. Nitta M, Okamura H, Aizawa S, Yamaizumi M (1997) Heat shock induces transient p53-dependent cell cycle arrest at G1/S. Oncogene 15: 561-568.

55. Nashimoto T, Komata T, Kanzawa T, Aoki H, Endo S, et al. (2005) Mild hyperthermia plus adenoviral p53over-expression additively inhibits the viability of human malignant glioma cells. Int J Hyperthermia 21: 615-629.

56. Fuse T, Yamada K, Asai K, Kato T, Nakanishi M (1996) Heat shock-mediated cell cycle arrest is accompanied by induction of p21 CKI. Biochem Biophys Res Commun 225: 759-763.

57. Madlener S, Rosner M, Krieger S, Giessrigl B, Gridling M, et al. (2009) Shor 42 degrees $C$ heat shock induces phosphorylation and degradation of Cdc25A which depends on p38MAPK, Chk2 and 14.3.3. Hum Mol Genet 18: 1990 2000

58. Furusawa Y, lizumi T, Fujiwara Y, Zhao QL, Tabuchi Y, et al. (2012) Inhibition of checkpoint kinase 1 abrogates G2/M checkpoint activation and promotes apoptosis under heat stress. Apoptosis 17: 102-112.

59. Tuul M, Kitao H, limori M, Matsuoka K, Kiyonari S, et al. (2013) Rad9, Rad17, TopBP1 and claspin play essential roles in heat-induced activation of ATR kinase and heat tolerance. PLoS One 8: e55361.

60. O'Connor MJ (2015) Targeting the DNA damage response in cancer. Mol Cell 60: $547-560$

61. Boissenot T, Bordat A, Fattal E, Tsapis N (2016) Ultrasound-triggered drug deliverly for cancer treatment using drug deliverly systems: From theoretical considerations to practical application. J Control Release 241: 144-163 